\title{
Stable transfection of protein kinase $C$ alpha cDNA in hormone-dependent breast cancer cell lines
}

D A Tonetti, M J Chisamore, W Grdina, H Schurz and V C Jordan

Correction to: British Journal of Cancer (2000) 83, 782-791. doi:10.1054/bjoc.2000.1326

The corresponding author of the above paper, Dr Debra Tonetti, has informed us that following Short Tandem Repeat analysis on all of their cell lines, the authors had discovered that the cell clone T47D:A18/ $\mathrm{PKC} \alpha$ is in fact MCF-7/PKC $\alpha$. This was verified using next-generation sequencing of the p53 mutation that is a hallmark of T47D cells. All other cell lines reported in this paper are as originally described. As both T47D and MCF-7 cells are oestrogen receptor (ER)positive breast cancer cell lines, the authors conclude that this error does not change the original interpretation of their work. The authors apologise for this error. 